\title{
openheart Combined biomarker testing for the prediction of left ventricular remodelling in ST-elevation myocardial infarction
}

\author{
Sebastian Johannes Reinstadler, ${ }^{1}$ Hans-Josef Feistritzer, ${ }^{1}$ Martin Reindl, ${ }^{1}$ \\ Gert Klug, ${ }^{1}$ Agnes Mayr, ${ }^{2}$ Johannes Mair, ${ }^{1}$ Werner Jaschke, ${ }^{2}$ Bernhard Metzler ${ }^{1}$
}

To cite: Reinstadler SJ, Feistritzer H-J, Reindl M, et al. Combined biomarker testing for the prediction of left ventricular remodelling in ST-elevation

myocardial infarction. Open Heart 2016;3:e000485. doi:10.1136/openhrt-2016000485

Received 9 June 2016 Revised 10 August 2016 Accepted 22 August 2016

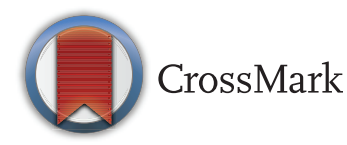

${ }^{1}$ Department of Cardiology and Angiology, University Clinic of Internal Medicine III, Medical University of Innsbruck, Innsbruck, Austria ${ }^{2}$ Department of Radiology, Medical University of Innsbruck, Innsbruck, Austria

\section{Correspondence to} Dr Bernhard Metzler; bernhard.metzler@tirolkliniken.at

\section{ABSTRACT \\ Objective: The utility of different biomarkers for the prediction of left ventricular remodelling (LVR) following ST-elevation myocardial infarction (STEMI) has been evaluated in several studies. However, very few data exist on the prognostic value of combined biomarkers. The aim of this study was to comprehensively investigate the prognostic value for LVR of routinely available biomarkers measured after reperfused STEMI.}

Methods: Serial measurements of N-terminal pro-Btype natriuretic peptide (NT-proBNP), high-sensitivity cardiac troponin T (hs-cTnT), aspartate aminotransferase (AST), alanine aminotransferase (ALT), lactate dehydrogenase (LDH) and highsensitivity $C$ reactive protein (hs-CRP) were performed in 123 patients with STEMI treated with primary percutaneous coronary intervention in this prospective observational study. Patients underwent cardiac MRI at 2 (1-4) and 125 (121-146) days after infarction. An increase in end-diastolic volume of $\geq 20 \%$ was defined as LVR.

Results: LVR occurred in 16 (13\%) patients. Peak concentrations of the following biomarkers showed significant areas under the curves (AUCs) for the prediction of LVR-NT-proBNP: $0.68(95 \% \mathrm{Cl} 0.59$ to $0.76, p=0.03$ ), hs-cTnT: 0.75 (95\% Cl 0.66 to 0.82 , p<0.01), AST: 0.72 (95\% Cl 0.63 to $0.79, p<0.01)$, ALT: 0.66 ( $95 \% \mathrm{Cl} 0.57$ to $0.75, p=0.03$ ), LDH: 0.78 $(95 \% \mathrm{Cl} 0.70$ to $0.85, p<0.01)$ and hs-CRP: $0.63(95 \%$ $\mathrm{Cl} 0.54$ to $0.72, p=0.05$ ). The combination of all biomarkers yielded a significant increase in AUC to 0.85 (95\% Cl 0.77 to 0.91 ) (all vs NT-proBNP: $p=0.02$, all vs hs-cTnT: $p=0.02$, all vs AST: $p<0.01$, all vs ALT: $p<0.01$, all vs hs-CRP: $p<0.01$ and all vs $L D H: p=0.04$ ) Conclusions: In patients with reperfused STEMI, the combined assessment of peak NT-proBNP, hs-cTnT, AST, ALT, hs-CRP and LDH provide incremental prognostic information for the prediction of LVR when compared with single-biomarker measurement.

\section{INTRODUCTION}

Outcome of patients suffering an ST-elevation myocardial infarction (STEMI)

\section{KEY QUESTIONS}

What is already known about this subject?

- The development of adverse left ventricular remodelling (LVR) is associated with heart failure and mortality in patients surviving acute ST-elevation myocardial infarction (STEMI). Determination of routine biomarkers during the acute phase after reperfusion therapy might be a simple method to estimate the risk of LVR for the individual patient. It is, however, unclear whether the combination of routine biomarkers improves risk stratification for LVR after STEMI.

What does this study add?

- This study demonstrates that peak concentrations of N-terminal pro-B-type natriuretic peptide, high-sensitivity cardiac troponin $\mathrm{T}$, aspartate aminotransferase, alanine transaminase, high-sensitivity $\mathrm{C}$ reactive protein and lactate dehydrogenase have significant ability to distinguish patients who develop LVR from those who do not. Importantly, the combination of all biomarkers provided incremental prognostic information for LVR when compared with single-biomarker testing.

How might this impact on clinical practice?

- Combined measurement of routine biomarkers improves early risk assessment for the development of LVR in patients with STEMI treated with primary percutaneous coronary intervention. Randomised studies are necessary to evaluate whether a biomarker-guided treatment strategy targeting LVR might improve patients outcome.

has markedly improved over the past decades. ${ }^{1}$ Nonetheless, survivors of the acute event are still at increased risk for further cardiovascular events. $^{2}$

Adverse left ventricular remodelling (LVR) is a dynamic process at tissue, cellular and molecular level, characterised by changes in ventricular geometry, stiffness and function, 
which affects the infarcted and non-infarcted myocardium. ${ }^{3}{ }^{4}$ It is well established that the degree of LVR is a major determinate of adverse clinical events after infarction. ${ }^{3-5}$ Therefore, early identification of patients at risk for LVR is regarded as an important step in post-STEMI risk stratification.

Biomarker measurement during the (sub-)acute phase after STEMI might be a simple and cost-effective tool to estimate the risk for LVR in clinical practice. To date, $>50$ biomarkers were described as potentially useful for the prediction of LVR. ${ }^{6}$ However, most of them were tested in relatively small number of patients $(<100$ participants) and only very few of them are available in daily clinical routine. Moreover, the potential incremental prognostic value for the combination of these biomarkers is largely unknown. ${ }^{7}$ Natriuretic peptides, cardiac troponins, inflammatory markers, transaminases and lactate dehydrogenase $(\mathrm{LDH})$ are often determined in the subacute phase after STEMI and associated with ejection fraction and myocardial damage,${ }^{8-12}$ which are major determinates of LVR. Moreover, they have been suggested for prognosis assessment in patients with STEMI. 8101113

Cardiac MRI (CMRI) provides high spatial and temporal resolution as well as high blood-tissue contrast and is therefore considered as the most accurate imaging technique to longitudinally assess changes in myocardial morphology and function after STEMI. ${ }^{14}$

Therefore, we aimed to investigate the prognostic value of routinely available biomarkers (N-terminal pro-B-type natriuretic peptide (NT-proBNP), high-sensitivity cardiac troponin $\mathrm{T}$ (hs-cTnT), aspartate aminotransferase (AST), alanine aminotransferase (ALT), LDH and high-sensitivity $\mathrm{C}$ reactive protein (hs-CRP)) measured during the (sub-)acute phase after STEMI, to predict CMRI-determined LVR. Moreover, we sought to evaluate a potential multimarker approach.

\section{METHODS}

\section{Study population}

One hundred and thirty-three consecutive patients presenting at our University Hospital with acute STEMI were enrolled in this prospective single-centre, observational study. Only patients presenting with first STEMI, as defined by the redefined ESC/ACG committee criteria, ${ }^{15}$ and treated with primary percutaneous coronary intervention (PPCI) were recruited. Exclusion criteria included age $<18$ years, history of a previous myocardial infarction or angiographically proven coronary artery disease, Killip-class III or IV, severe renal failure (defined as an estimated glomerular filtration rate $<30 \mathrm{~mL} / \mathrm{min} / 1.73 \mathrm{~m}^{2}$ ) or contraindications for CMRI. Patient demographics and medications were prospectively obtained during hospitalisation for the index event and at 4 months follow-up using a standardised questionnaire. Furthermore, all patients underwent physical examination. The study was approved by the local ethic committee of Medical University of Innsbruck and written informed consent was obtained from each participant prior to inclusion in the study.

\section{Cardiac MRI}

Patients underwent CMRI on a $1.5 \mathrm{~T}$ scanner (Magnetom AVANTO-scanner, Siemens, Erlangen, Germany) within the first week and 4 months post-PPCI for STEMI. The CMRI protocol and postprocessing was published in detail previously. ${ }^{14}$ Briefly, cine CMRI images in short axis (10-12 slices, covering the heart from the base to the apex) were obtained using breathhold, retrospective ECG-triggered trueFISP bright-blood sequences. Evaluation of cine images was performed using commercially available software (ARGUS, Siemens). Late enhancement CMRI images were obtained $10 \mathrm{~min}$ after a bolus injection of gadolinium $(0.1 \mathrm{mmol} / \mathrm{kg})$ by applying an ECG-triggered phasesensitive inversion recovery single-shot trueFISP sequence with consecutive short-axis slices. Infarct characteristics were evaluated as reported previously. ${ }^{16}$ An increase in end-diastolic volume of $20 \%$ or more between baseline and follow-up CMRI investigation was defined as adverse remodelling. ${ }^{16}$

\section{Biomarker testing}

Venous blood samples were obtained on admission and subsequently once daily from day 1 to day 4 after PPCI. hs-cTnT was additionally measured 6 and 12 hours post-PCI. All samples were immediately analysed at the central laboratory of the University Hospital of Innsbruck by personnel blinded to study data. NT-proBNP concentrations were calculated using a commercially available assay with an E170 instrument (proBNP II assay using monoclonal antibodies on a Modular, Roche Diagnostics, Vienna, Austria). The analytical limit of detection of NT-proBNP is $5 \mathrm{ng} / \mathrm{L}$ and the limit of quantification is $50 \mathrm{ng} / \mathrm{L}$. The intra-assay coefficient of variations $(\mathrm{CV})$ are $1.9 \%$ at a concentration of $64 \mathrm{ng} / \mathrm{L}$ and $1.2 \%$ at a concentration of $2105 \mathrm{ng} / \mathrm{L}$, and the interassay $\mathrm{CV}$ are $3.1 \%$ at a concentration of $46 \mathrm{ng} / \mathrm{L}$ and $2.7 \%$ at a concentration of $2170 \mathrm{ng} / \mathrm{L}$ according to the package insert. hs-cTnT concentrations were also measured using an assay with an E170 instrument (Roche Diagnostics). ${ }^{17}$ The analytical limit of detection and the 99th centile upper reference limit are 5 and $14 \mathrm{ng} / \mathrm{L}$, respectively. The $\mathrm{CV}$ value is $<10 \%$ at $13 \mathrm{ng} / \mathrm{L}$. LDH activities, AST and ALT concentrations as well as hs-CRP concentrations were measured by routine assays as described previously. ${ }^{9}$ Peak levels of each biomarker were calculated.

\section{Statistical analysis}

Distribution of continuous variables was tested with the Shapiro-Wilk test. Results of continuous variables are expressed as mean \pm SD or median with IQR. Results of categorical variables are shown as number and corresponding percentages. The Pearson or Spearman rank 
correlation was calculated as indicated. Differences in location between groups were tested accordingly by means of the t-test (Welch) and the Wilcoxon-Mann-Whitney U test. Proportions were compared by $\chi^{2}$ test and Fisher's exact test as indicated. The utility of biomarkers for the prediction of LVR was tested with the use of receiver operating characteristic (ROC) analysis. The area under the curve (AUC), sensitivity and specificity were calculated. C-statistics according to DeLong $e t a l^{18}$ were applied to evaluate the combined prognostic value in predicting LVR. A two-sided $\alpha=5 \%$ was determined overall. We used SPSS Statistics V.22.0.0.1 (IBM Corp., Armonk, New York, USA) and MedCalc V.15.8 (MedCalc Software, Ostend, Belgium) for statistical analysis.

\section{RESULTS}

\section{Baseline characteristics and CMRI findings}

Of 133 enrolled patients with STEMI, 10 (92.5\%) patients had no complete CMRI evaluation and/or biomarker sampling and were therefore excluded. Thus, data of $123(92.5 \%)$ patients were available for the analysis (figure 1). Table 1 shows the baseline characteristics (including biomarker concentrations) of the entire study cohort as well as for patients with and without LVR. There was no significant correlation between pain-to-balloon time and the investigated biomarkers (all p >0.05). All patients underwent CMRI 2 (1-4) and 125 (121-146) days after STEMI. LVR occurred in 16 patients $(13 \%)$ at 4 months follow-up. CMRI results at baseline and follow-up are summarised in table 2. Of note, only patients without LVR showed a significant increase in LVEF $(p<0.01)$, whereas patients with LVR had no significant improvement in LVEF $(p=0.20)$. Baseline infarct size was larger in the LVR group $(\mathrm{p}<0.01)$, but significantly decreased in both groups from baseline to follow-up (all $\mathrm{p}<0.05)$. The presence of microvascular obstruction was also significantly more likely in the LVR group $(\mathrm{p}=0.02)$.

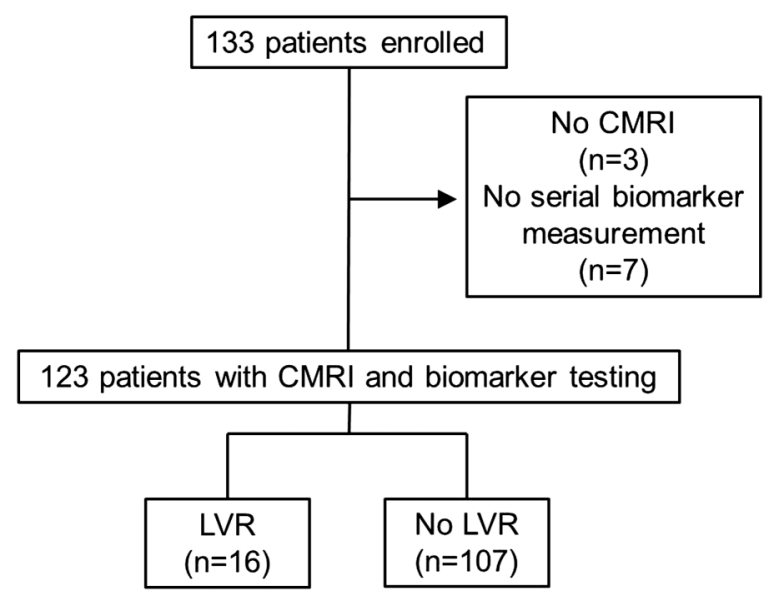

Figure 1 Flow diagram of study patients. CMRI, cardiac MRI, LVR, left ventricular remodelling.

\section{Biomarkers and LV remodelling}

Patients with LVR had higher peak concentrations of NT-proBNP (1385 (616-3949) ng/L vs 677 (151-1267), $\mathrm{p}=0.02)$, hs-cTnT (10 153 (5722-15 995) ng/L vs 4709 (1985-8025), $\mathrm{p}<0.01)$, AST (375 (240-646) U/L vs 231 (126-349), $\mathrm{p}<0.01)$, ALT (73 (49-141) U/L vs 52 (3881), $\mathrm{p}=0.04)$ and $\mathrm{LDH}(917(654-1530) \mathrm{U} / \mathrm{L}$ vs 523 (324-791), $\quad \mathrm{p}<0.01)$ when compared with patients without LVR. The predictive value of biomarkers for LVR was assessed by ROC analysis (figure 2). The AUCs of significant predictors were 0.68 (95\% CI 0.52 to 0.83 , $\mathrm{p}=0.03$ ) for NT-proBNP, 0.75 (95\% CI 0.62 to 0.87 , $\mathrm{p}<0.01$ ) for hs-cTnT, 0.72 (95\% CI 0.59 to $0.84, \mathrm{p}<0.01$ ) for AST, 0.66 (95\% CI 0.57 to $0.75, \mathrm{p}=0.03$ ) for ALT, 0.63 (95\% CI 0.54 to $0.72, \mathrm{p}=0.05)$ for hs-CRP and $0.78(95 \%$ CI 0.67 to $0.90, \mathrm{p}<0.01$ ) for $\mathrm{LDH}$. Cut-off values with highest sensitivity and specificity of biomarkers are shown in table 3 . The combination of all significant biomarkers in addition to NT-proBNP resulted in a significant increase in the c-statistics from 0.68 (95\% CI 0.59 to 0.76 ) to 0.85 (95\% CI 0.77 to $0.91, \mathrm{p}=0.02$ ), thus showing an incremental predictive value of combined biomarker assessment over NT-proBNP alone. Furthermore, the combination of biomarkers in addition to hs-cTnT $(\mathrm{p}=0.02)$, AST $(\mathrm{p}<0.01)$, ALT $(\mathrm{p}<0.01)$, hs-CRP $(p<0.01)$ and LDH $(p=0.04)$ also resulted in a significant increase in the c-statistics.

In contrast to peak concentrations, admission concentrations of all investigated biomarkers were not significantly different between patients who developed LVR compared with patients who did not develop LVR (table 1). In line with this, none of these biomarkers showed a significant discriminatory ability for the prediction of LVR on admission (AUC of NT-proBNP $=0.57$, AUC of hs-cTnT $=0.54$, AUC of AST $=0.55$, AUC of ALT $=0.55$, AUC of $\mathrm{LDH}=0.59$ and AUC of hs-CRP=0.44, all $\mathrm{p}>0.05$ ).

\section{DISCUSSION}

This study sought to comprehensively investigate the value of routine biomarkers for the prediction of LVR after reperfused STEMI. Peak concentrations of NT-proBNP, hs-cTnT, AST, ALT, hs-CRP and LDH, measured during the (sub-)acute phase after PPCI, showed significant ability to distinguish patients who developed LVR from those who did not. Importantly, the combination of all six biomarkers provided incremental prognostic information on LVR when compared with single-biomarker testing. In contrast, admission values of the investigated biomarkers were not useful for the prediction of LVR. Together, our data highlight the use of a multimarker approach for the prediction of LVR which should be evaluated in further larger trials.

STEMI is one of the main causes of heart failure and LVR plays a central role in this process. LVR rapidly occurs following STEMI, and it is well established that progressive postinfarction remodelling is an important predictor of poor clinical outcome, including mortality. ${ }^{34}$ 
Table 1 Baseline patient characteristics

\begin{tabular}{|c|c|c|c|c|}
\hline Variables & $\begin{array}{l}\text { All patients } \\
(n=123)\end{array}$ & $\begin{array}{l}\text { LV remodelling } \\
(n=16)\end{array}$ & $\begin{array}{l}\text { No LV remodelling } \\
(n=107)\end{array}$ & p Value \\
\hline Age, years & $57 \pm 11$ & $60 \pm 11$ & $57 \pm 10$ & 0.24 \\
\hline Male sex, n (\%) & $108(88)$ & $12(75)$ & $96(90)$ & 0.09 \\
\hline Body mass index, $\mathrm{kg} / \mathrm{m}^{2}$ & $26(25-28)$ & $27(25-30)$ & $26(25-28)$ & 0.52 \\
\hline \multicolumn{5}{|l|}{ Cardiovascular risk factors } \\
\hline Hypertension, n (\%) & $77(63)$ & $12(75)$ & $65(61)$ & 0.27 \\
\hline Diabetes mellitus, n (\%) & $10(8)$ & $0(0)$ & $10(9)$ & 0.20 \\
\hline Current smoker, n (\%) & $63(51)$ & $7(44)$ & $56(52)$ & 0.52 \\
\hline Hypercholesterolaemia, n (\%) & $78(63)$ & $8(50)$ & $70(65)$ & 0.23 \\
\hline Family history for AMI, n (\%) & $35(29)$ & $4(25)$ & $31(29)$ & 0.74 \\
\hline Pain-to-balloon time, $\min$ & $192(137-352)$ & $214(132-421)$ & $192(137-351)$ & 0.87 \\
\hline Infarct-related artery & & & & 0.03 \\
\hline Right coronary artery, n (\%) & $58(47)$ & $5(31)$ & $53(49)$ & \\
\hline Left anterior descending artery, $\mathrm{n}(\%)$ & $50(41)$ & $11(69)$ & $39(36)$ & \\
\hline Left circumflex coronary artery, $\mathrm{n}(\%)$ & $15(12)$ & $0(0)$ & $15(14)$ & \\
\hline Number of diseased vessels & & & & 0.09 \\
\hline $1, \mathrm{n}(\%)$ & $64(52)$ & $9(56)$ & $55(51)$ & \\
\hline $2, \mathrm{n}(\%)$ & $44(36)$ & $3(18)$ & $41(38)$ & \\
\hline $3, \mathrm{n}(\%)$ & $15(12)$ & $4(25)$ & $11(10)$ & \\
\hline \multicolumn{5}{|l|}{ Medication at discharge } \\
\hline Acetylsalicylic acid, $\mathrm{n}(\%)$ & $123(100)$ & $16(100)$ & $107(100)$ & 1.0 \\
\hline$\beta$-Blocker, n (\%) & $104(85)$ & $14(87)$ & $90(84)$ & 0.99 \\
\hline ACE-I or ARB, $n(\%)$ & $105(86)$ & $14(87)$ & $91(85)$ & 0.41 \\
\hline Statin, $\mathrm{n}(\%)$ & $117(95)$ & $16(100)$ & $101(94)$ & 0.57 \\
\hline Admission NT-proBNP, ng/L & $100(58-401)$ & $125(63-838)$ & $99(54-371)$ & 0.44 \\
\hline Admission hs-cTnT, ng/L & $144(21-1835)$ & $132(18-10263)$ & $153(25-1617)$ & 0.58 \\
\hline Admission AST, U/L & $74(33-223)$ & $97(36-410)$ & $73(33-213)$ & 0.55 \\
\hline Admission ALT, U/L & $37(27-65)$ & $37(28-90)$ & $36(27-65)$ & 0.50 \\
\hline Admission LDH, U/L & $237(196-343)$ & $241(204-586)$ & $234(196-324)$ & 0.27 \\
\hline Admission hs-CRP, mg/L & $2.1(1.1-5.0)$ & $1.8(1.1-3.6)$ & $2.1(1.1-5.6)$ & 0.41 \\
\hline Peak NT-proBNP, ng/L & $704(196-1391)$ & $1385(616-3949)$ & $677(151-1267)$ & 0.02 \\
\hline Peak hs-cTnT, ng/L & $5464(2337-8574)$ & 10153 (5722-15 995) & $4709(1985-8025)$ & $<0.01$ \\
\hline Peak AST, U/L & $245(138-369)$ & $375(240-646)$ & $231(126-349)$ & $<0.01$ \\
\hline Peak ALT, U/L & $57(40-88)$ & $73(49-141)$ & $52(38-81)$ & 0.04 \\
\hline Peak LDH, U/L & $593(353-832)$ & $917(654-1530)$ & $523(324-791)$ & $<0.01$ \\
\hline Peak hs-CRP, mg/L & $19.9(9.2-44.4)$ & $31.8(16.7-56.4)$ & $17.0(9.0-38.8)$ & 0.08 \\
\hline
\end{tabular}

Table 2 Cardiovascular MRI results

\begin{tabular}{|c|c|c|c|}
\hline Characteristics & Baseline & Follow-up & p Value \\
\hline LVEF (\%) & $55(49-61)$ & $60(54-66)$ & $<0.01$ \\
\hline Infarct size (\%LVMM) & $16(9-25)$ & $11(6-16)$ & $<0.01$ \\
\hline LV stroke volume (mL) & 79 (68-92) & $88(80-99)$ & $<0.01$ \\
\hline LV end-diastolic volume (mL) & $150(128-166)$ & $152(128-170)$ & 0.05 \\
\hline LV end-systolic volume (mL) & $68(50-81)$ & $58(47-75)$ & $<0.01$ \\
\hline Microvascular obstruction: $n(\%)$ & $65(52)$ & NA & NA \\
\hline
\end{tabular}

Data are presented as median plus IQR.

LV, left ventricular; LVEF, left ventricular ejection fraction; LVMM, left ventricular myocardial mass; NA, not applicable.

In the current study, LVR occurred in $\sim 13 \%$ of patients. This is in line with other recent reports that found a similar rate of LVR in comparable patient's cohorts. 71920 Patients were largely treated according to recommended therapy, including a high use of ACE inhibitors and $\beta$-blockers. Our results therefore underline that even in the modern era of PPCI and upstream heart failure therapy, LVR still occurs in a significant proportion of patients. Consequently, LVR remains a major clinical problem in the current era of STEMI management and 


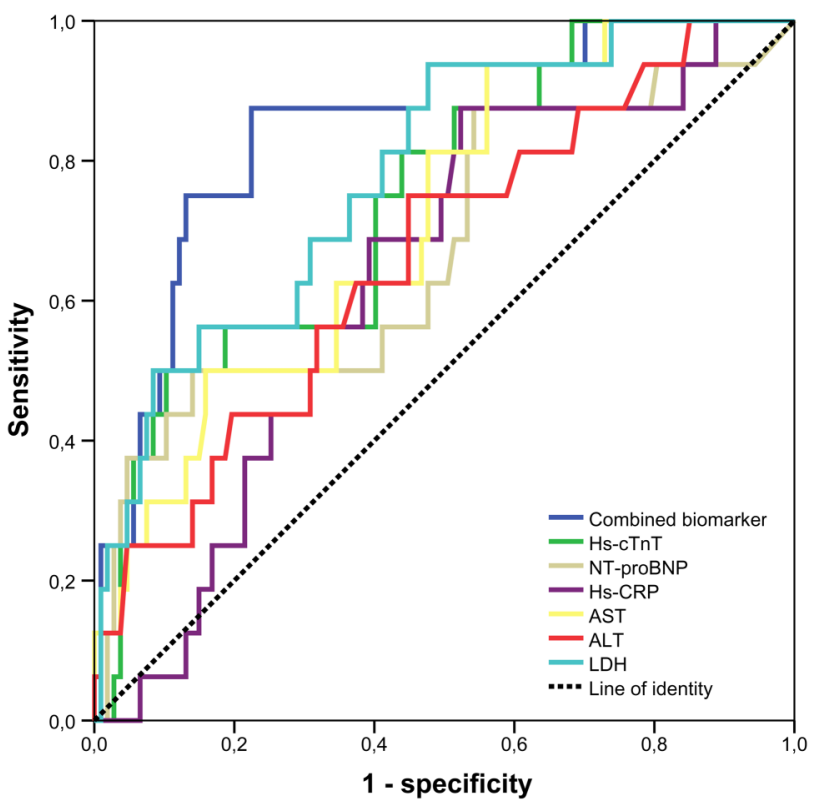

Figure 2 ROC curve analysis for the prediction of LVR by investigated routine biomarkers and their combination. NT-proBNP, N-terminal pro B-type natriuretic peptide, hs-cTnT, high-sensitivity cardiac troponin T, AST, aspartate aminotransferase, ALT, alanine transaminase, hs-CRP, high-sensitivity $\mathrm{C}$ reactive protein, $\mathrm{LDH}$, lactate dehydrogenase, LVR, left ventricular remodelling; ROC, receiver operating characteristic.

better identification of patients at risk for LVR is essential to guide treatment and follow-up. Biomarkers may allow such an early prediction of LVR that could easily be used in a broad range of patients with STEMI. Several biomarkers, including natriuretic peptides, ${ }^{13}$ cardiac troponins, ${ }^{10}$ inflammatory markers, ${ }^{21}$ transaminases ${ }^{11}$ and $\mathrm{LDH}_{,}{ }^{22}$ are readily available, are routinely measured in patients hospitalised for STEMI and their measurement is relatively cheap. These biomarkers have been described to be associated with myocardial function, infarct size and microvascular injury, ${ }^{9-12} 23$ which are known determinants of LVR after STEMI. Most of them, but not all (transaminases and LDH), have also been investigated for their usefulness in predicting the occurrence of LVR after myocardial infarction. ${ }^{6}$ However, these studies were hampered by several limitations, including small numbers of included patients (most enrolled $<100$ patients) ${ }^{6}$ the inclusion of highly selected patient groups (eg, patients with anterior Q-wave MI only) ${ }^{24}$ and the use of echocardiography rather than CMRI, ${ }^{6} 724$ which is the reference standard for the assessment of LVR. ${ }^{14}$ Finally, studies comparing the predictive value of multiple biomarkers within the same study are missing. Thus, to the best of our knowledge, this is the first study to investigate a multimarker approach for the prediction of CMRI-determined LVR after PPCI for STEMI. We found that peak concentrations of NT-proBNP, hs-cTnT, AST, ALT hs-CRP and LDH were significant predictors of LVR. Therefore, we not only confirm the significant relation of NT-proBNP, hs-cTnT and hs-CRP with LVR in a large cohort of patients with STEMI, but also show that AST, ALT and LDH are useful for the prediction of LVR in this setting. Importantly, we also observed that the combined measurement of these biomarkers might further increase the discriminative capacity. Our results therefore suggest that combining these biomarkers could constitute a strong tool for refining risk stratification after STEMI. This could have therapeutic implications, since patients at high risk for LVR could be treated more aggressively and followed more closely. In the light of the lack of trials, however, designed to prospectively investigate the value of a biomarker-guided treatment strategy, their use for risk stratification of the postinfarction patient awaits further validation and research.

Conclusions on the pathophysiological associations between investigated biomarkers and LVR as well as specific processes leading to their elevation are beyond the scope of this study, but the following is appreciable to mention. In patients with acute MI, AST, ALT and LDH are both related with adverse clinical outcome, even in the contemporary era of mechanical reperfusion. ${ }^{25} 26$ There is recent evidence showing that circulating levels of AST, ALT and LDH are not only an indicator of infarct size, but may also reflect more extensive microvascular injury in reperfused STEMI. ${ }^{9}{ }^{11}$ Infarct size and microvascular injury are strong predictors of $\mathrm{LVR}^{27}$ and subsequently adverse clinical outcome. ${ }^{28} 29$ Although most evidence indicate that AST and ALT release is primarily of myocardial origin, circulatory failure with subsequent hypoxic live injury might in part also explain these elevations. ${ }^{8}$ In particular, AST was superior to cardiac troponin I in predicting outcome in STEMI in this study.

Table 3 Predictive accuracy of routine biomarkers for left ventricular remodelling

\begin{tabular}{lllll}
\hline Biomarker & AUC & Cut-off concentration & Sensitivity (\%) & Specificity (\%) \\
\hline NT-proBNP & $0.68(0.59-0.76)$ & $1931 \mathrm{ng} / \mathrm{L}$ & 50 & 86 \\
hs-cTnT & $0.75(0.66-0.82)$ & $5919 \mathrm{ng} / \mathrm{L}$ & 75 & 60 \\
AST & $0.72(0.63-0.79)$ & $300 \mathrm{U} / \mathrm{L}$ & 63 & 65 \\
ALT & $0.66(0.57-0.75)$ & $85 \mathrm{U} / \mathrm{L}$ & 75 & 58 \\
hs-CRP & $0.63(0.54-0.72)$ & $22.5 \mathrm{mg} / \mathrm{L}$ & 70 & 48 \\
LDH & $0.78(0.70-0.85)$ & $757 \mathrm{U} / \mathrm{L}$ & 71 \\
\hline
\end{tabular}

ALT, alanine transaminase; AST, aspartate aminotransferase; AUC, area under the curve; hs-CRP, high-sensitivity $\mathrm{C}$ reactive protein; hs-cTnT, high-sensitivity cardiac troponin T; LDH, lactate dehydrogenase; LVR, left ventricular remodelling; NT-proBNP, N-terminal pro B-type natriuretic peptide. 
Although, it is well established that cardiac troponins are useful for the prediction of LVR, ${ }^{6}{ }^{30}$ there are no studies that used high-sensitivity troponin assays. The introduction of hs-cTnT assays not only resulted in a significant increase in the sensitivity of cardiac troponins at an early diagnostic stage, but might also improve risk stratification. ${ }^{31}$ Nonetheless, we found no association between admission hs-cTnT and LVR in the current analysis. On the other hand, peak hs-cTnT was confirmed to be significantly related with LVR. NT-proBNP, which is released following increased myocardial wall stress, is mainly used as an indicator of heart failure progression. ${ }^{32}$ Our study underscores that NT-proBNP is also useful for the prediction of LVR, a finding which is consistent with several other investigations, ${ }^{6}$ such as the study by Orn et al. ${ }^{33}$ Finally, the degree of inflammation, as reflected by circulating hs-CRP, is associated with LVR. ${ }^{7}$ Our finding of a prognostic significance of circulating hs-CRP for the prediction of LVR is in line with these previous results.

Our study has several limitations. Although this study represents the largest CMRI trial on the prognostic value of combined routine biomarkers for LVR after STEMI so far, further confirmation in larger studies is very important. Moreover, the potential impact of several important determinants of LVR (eg, microvascular obstruction or time to treatment) on the predictive value of biomarkers could not be investigated in the current trial. These specific issues also need clarification in larger investigations. As most other studies, we preferred peak concentrations over AUC values since the first approach is a much more feasible measurement in daily clinical routine. Inclusion and exclusion criteria might limit generalisation of study results, as, for example, patients presenting with Killip class $\geq 3$ were not eligible for this study. Nevertheless, patient and CMRI characteristics are comparable with other larger multicentre CMRI STEMI trials. ${ }^{28}$ Finally, there is a plethora of other potential useful biomarkers for the prediction of LVR, ${ }^{6}$ which are, however, not available in daily routine and were therefore not incorporated in the current analysis.

In conclusion, we found that peak concentrations of NT-proBNP, hs-cTnT, AST, ALT, hs-CRP and LDH after PPCI for STEMI provide prognostic information on the development of LVR as visualised by CMRI. Combined sampling of these routinely available biomarkers could facilitate the identification of patients at increased risk of LVR that might benefit from more intensive postinfarction care. However, considering the lack of randomised trials evaluating a biomarker-guided treatment strategy, this issue awaits further validation and research.

Acknowledgements This study was supported by grants from the 'Austrian Society of Cardiology' as well as by the intramural funding programme of Medical University Innsbruck for young scientists MUI-START, 'Project 2015-06-013'.

Contributors SJR designed the study; analysis and interpretation of data; drafting of the manuscript. H-JF contributed to interpretation of the data; drafting of the manuscript and revising critically for important intellectual content. MR contributed to analysis and interpretation of the data; revising critically for important intellectual content. GK contributed to analysis and interpretation of the data; revising critically for important intellectual content. AM contributed to image analysis; revising critically for important intellectual content. JM contributed to interpretation of the data; revising critically for important intellectual content. WJ contributed to interpretation of the data; revising critically for important intellectual content. BM (corresponding author) designed the study; analysis and interpretation of data; drafting of the manuscript; revising critically for important intellectual content.

Funding Austrian Society of Cardiology, Medical University Innsbruck "MUI-START" (Project 2015-06-013)

Competing interests None declared.

Provenance and peer review Not commissioned; externally peer reviewed.

Data sharing statement No additional data are available.

Open Access This is an Open Access article distributed in accordance with the Creative Commons Attribution Non Commercial (CC BY-NC 4.0) license, which permits others to distribute, remix, adapt, build upon this work noncommercially, and license their derivative works on different terms, provided the original work is properly cited and the use is non-commercial. See: http:// creativecommons.org/licenses/by-nc/4.0/

\section{REFERENCES}

1. McManus DD, Gore J, Yarzebski J, et al. Recent trends in the incidence, treatment, and outcomes of patients with STEMI and NSTEMI. Am J Med 2011;124:40-7.

2. Jernberg $\mathrm{T}$, Johanson $\mathrm{P}$, Held $\mathrm{C}$, et al. Association between adoption of evidence-based treatment and survival for patients with ST-elevation myocardial infarction. JAMA 2011;305:1677-84

3. Cohn JN, Ferrari R, Sharpe N. Cardiac remodeling-concepts and clinical implications: a consensus paper from an international forum on cardiac remodeling. Behalf of an International Forum on Cardiac Remodeling. J Am Coll Cardiol 2000;35:569-82.

4. Altara R, Manca M, Sabra R, et al. Temporal cardiac remodeling post-myocardial infarction: dynamics and prognostic implications in personalized medicine. Heart Fail Rev 2015:21;25-47.

5. Kramer DG, Trikalinos TA, Kent DM, et al. Quantitative evaluation of drug or device effects on ventricular remodeling as predictors of therapeutic effects on mortality in patients with heart failure and reduced ejection fraction: a meta-analytic approach. J Am Coll Cardiol 2010;56:392-406.

6. Fertin M, Dubois E, Belliard A, et al. Usefulness of circulating biomarkers for the prediction of left ventricular remodeling after myocardial infarction. Am J Cardiol 2012;110:277-83.

7. Urbano-Moral JA, Lopez-Haldon JE, Fernandez M, et al. Prognostic value of different serum biomarkers for left ventricular remodelling after ST-elevation myocardial infarction treated with primary percutaneous coronary intervention. Heart 2012;98:1153-9.

8. Moon J, Kang W, Oh PC, et al. Serum transaminase determined in the emergency room predicts outcomes in patients with acute ST-segment elevation myocardial infarction who undergo primary percutaneous coronary intervention. Int J Cardiol 2014;177:442-7.

9. Mayr A, Klug G, Schocke M, et al. Late microvascular obstruction after acute myocardial infarction: relation with cardiac and inflammatory markers. Int J Cardiol 2012;157:391-6.

10. Hall TS, Hallén J, Krucoff MW, et al. Cardiac troponin I for prediction of clinical outcomes and cardiac function through 3-month follow-up after primary percutaneous coronary intervention for ST-segment elevation myocardial infarction. Am Heart J 2015;169:257-65.e1.

11. Reinstadler SJ, Reindl M, Feistritzer $\mathrm{HJ}$, et al. Prognostic significance of transaminases after acute ST-elevation myocardial infarction: insights from a cardiac magnetic resonance study. Wien Klin Wochenschr 2015;127:843-50.

12. Reindl M, Reinstadler SJ, Feistritzer HJ, et al. Relation of inflammatory markers with myocardial and microvascular injury in patients with reperfused ST-elevation myocardial infarction. Eur Heart J Acute Cardiovasc Care 2016. Published Online First: 20 Jul 2016. doi:2048872616661691

13. Helanova K, Littnerova S, Kubena $P$, et al. Prognostic impact of neutrophil gelatinase-associated lipocalin and B-type natriuretic in patients with ST-elevation myocardial infarction treated by primary PCl: a prospective observational cohort study. BMJ Open 2015;5: e006872.

14. Klug G, Metzler B. Assessing myocardial recovery following ST-segment elevation myocardial infarction: short- and long-term perspectives using cardiovascular magnetic resonance. Expert Rev Cardiovasc Ther 2013;11:203-19. 
15. Thygesen K, Alpert JS, Jaffe AS, et al. Third universal definition of myocardial infarction. Eur Heart J 2012;33:2551-67.

16. Reinstadler SJ, Klug G, Feistritzer HJ, et al. Association of copeptin with myocardial infarct size and myocardial function after ST segment elevation myocardial infarction. Heart 2013;99:1525-9.

17. Feistritzer HJ, Klug G, Reinstadler SJ, et al. Aortic stiffness is associated with elevated high-sensitivity cardiac troponin $\mathrm{T}$ concentrations at a chronic stage after ST-segment elevation myocardial infarction. J Hypertens 2015;33:1970-6.

18. DeLong ER, DeLong DM, Clarke-Pearson DL. Comparing the areas under two or more correlated receiver operating characteristic curves: a nonparametric approach. Biometrics 1988;44:837-45.

19. Feistritzer HJ, Klug G, Reinstadler SJ, et al. Fetuin-A is related to infarct size, left ventricular function and remodelling after acute STEMI. Open Heart 2015;2:e000244.

20. Dominguez-Rodriguez A, Abreu-Gonzalez P, Avanzas P, et al. Neopterin predicts left ventricular remodeling in patients with ST-segment elevation myocardial infarction undergoing primary percutaneous coronary intervention. Atherosclerosis 2010;211:574-8.

21. Seropian IM, Sonnino C, Van Tassell BW, et al. Inflammatory markers in ST-elevation acute myocardial infarction. Eur Heart $J$ Acute Cardiovasc Care 2016;5:382-95.

22. Sjauw KD, van der Horst IC, Nijsten MW, et al. Value of routine admission laboratory tests to predict thirty-day mortality in patients with acute myocardial infarction. Am J Cardiol 2006;97:1435-40.

23. Reinstadler SJ, Feistritzer HJ, Klug G, et al. High-sensitivity troponin $T$ for prediction of left ventricular function and infarct size one year following ST-elevation myocardial infarction. Int J Cardiol 2016;202:188-93.

24. Fertin M, Hennache B, Hamon M, et al. Usefulness of serial assessment of B-type natriuretic peptide, troponin I, and C-reactive protein to predict left ventricular remodeling after acute myocardial infarction (from the REVE-2 study). Am J Cardiol 2010;106:1410-16.

25. Lofthus DM, Stevens SR, Armstrong PW, et al. Pattern of liver enzyme elevations in acute ST-elevation myocardial infarction. Coron Art Dis 2012;23:22-30.

26. Wei S, Mao L, Liu B, et al. Serum biomarkers and the prognosis of AMI patients. Herz 2014;39:384-9.

27. Mather AN, Fairbairn TA, Ball SG, et al. Reperfusion haemorrhage as determined by cardiovascular MRI is a predictor of adverse left ventricular remodelling and markers of late arrhythmic risk. Heart 2011:97:453-9.

28. Eitel I, de Waha S, Wöhrle J, et al. Comprehensive prognosis assessment by CMR imaging after ST-segment elevation myocardia infarction. J Am Coll Cardiol 2014;64:1217-26.

29. Reinstadler SJ, Baum A, Rommel KP, et al. ST-segment depression resolution predicts infarct size and reperfusion injury in ST-elevation myocardial infarction. Heart 2015;101:1819-25.

30. Hallén J, Jensen JK, Fagerland MW, et al. Cardiac troponin I for the prediction of functional recovery and left ventricular remodelling following primary percutaneous coronary intervention for ST-elevation myocardial infarction. Heart 2010;96:1892-7.

31. Mair J. High-sensitivity cardiac troponins in everyday clinical practice. World J Cardiol 2014;6:175-82.

32. van der Velde AR, Meijers WC, de Boer RA. Biomarkers for risk prediction in acute decompensated heart failure. Curr Heart Fail Rep 2014;11:246-59.

33. Orn S, Manhenke C, Squire IB, et al. Plasma MMP-2, MMP-9 and $\mathrm{N}$-BNP in long-term survivors following complicated myocardial infarction: relation to cardiac magnetic resonance imaging measures of left ventricular structure and function. J Card Fail 2007;13:843-9. 